\title{
14. MIOCENE CORBISEMA TRIACANTHA ZONE PHYTOPLANKTON FROM DEEP SEA DRILLING PROJECT SITES 415 AND 416, OFF NORTHWEST AFRICA
}

\author{
David Bukry, U.S. Geological Survey, Scripps Institution of Oceanography, La Jolla, California
}

\section{SUMMARY}

Cores 415-4 and 415-5 and Cores 416A-1 and 416A-2, from sites off the northwest coast of Africa (Figure 1), contain diverse Miocene coccoliths, diatoms, and silicoflagellates. All the cores contain silicoflagellate assemblages of the middle- and lower-Miocene Corbisema triacantha Zone (Figure 2). One sample from the bottom of Core 415-5 may belong to a new Naviculopsis ponticula Zone. Coccoliths of the lower- or middleMiocene Helicosphaera ampliaperta Zone occur in Cores 415-5 and 416A-2; the next higher Sphenolithus heteromorphus Zone occurs in Cores 415-4 and 416A-1. Diatom zones are not identified from the assemblages (Figure 3), but the presence of short-ranged Annellus californicus and Raphidodiscus marylandicus suggests a diatom correlation near the boundary between lower and middle Miocene (Andrews, 1974; Ryan et al., 1974; Schrader and Fenner, 1976).

\section{SILICOFLAGELLATES}

Lower and middle Miocene silicoflagellates are common and well preserved at Sites 415 and 416 . Like the associated diatoms they are restricted to Cores 4 and 5 in Hole 415 and Cores 1 and 2 in Hole 416A. Siliceous microfossils reported in Core 3 at Hole 416A are mainly sponge spicules - mostly tylostyles (Hyman, 1940, fig. $81-3$; Bukry, 1978, pl. 13, figs. 10,11, 13) broken into 20 to $30 \mu \mathrm{m}$ lengths in Sample 416A-3-1, 116-117 cm (451 m).

The silicoflagellate assemblages of Sites 415 and 416 differ from those reported at nearby Sites 369 and 370 mainly in changes in relative abundances of species, such as the greater numbers of Dictyocha brevispina ausonia. The association of this taxon with early forms of D. pulchella (normal and deflandroid) and $M$. elliptica s. str. is characteristic of the Corbisema triacantha Zone in this region (Bukry, 1977). The lower-Miocene assemblages of Core 416A-2 are distinguished from all the others by the exclusive occurrence of the naviculoid variant (Deflandre, 1941) of D. brevispina ausonia. A majority of these variants have completely lost their minor axis portals but retain the bar and minor axis spines. Transitional specimens of $D$. brevispina ausonia having unusually small portals are present. The variants lack basal pikes and are similar in size and shape to associated Mesocena elliptica, suggesting that the exceptional variability of the local $D$. brevispina ausonia group might be a source for polyphyletic Mesocena. The exact form of this variant was first illustrated by Ehrenberg (1854; as Dictyocha pons) from Oran, Algeria, and later by Ling (1972; as Dictyocha ausonia) from Trinidad in the lower-Miocene Globigerinatella insueta Zone. This provides accurate correlation with Hole 416A, because Bramlette and Wilcoxon (1967) studied the coccoliths in the same "radiolarian-rich" locality from the $G$. insueta Zone in Trinidad and established the Helicosphaera ampliaperta Zone. The same coccolith zone is associated with the common naviculoid $D$. brevispina ausonia at Hole 416A. Therefore, this silicoflagellate is more than a local variant and has a regional range across the subtropical North Atlantic from Trinidad to Algeria. Its stratigraphic range must be short, because it was not encountered in the parts of the Naviculopsis lata Zone and Corbisema triacantha Zone that were sampled at nearby Sites 369 and 370 . The absence of guide fossil Distephanus stauracanthus from the ocean-margin setting that was studied at Sites 415 and 416 supports the conclusion of Ernissee et al. (1977) that this species is restricted to the middle Miocene in the Atlantic basin.

Naviculopsis navicula and $N$. ponticula occur together in the bottom sample from Core 415-5 and suggest that the sample could be assigned to a new lower-Miocene Naviculopsis ponticula Zone on the basis of the presence of these two species and the absence of $N$. quadrata. This is tentative, because the relative ranges of Naviculopsis in the upper lower Miocene are not established.

Silicoflagellate taxonomy follows that in earlier DSDP volumes, especially volumes 41 and 44 (Bukry, 1978a, 1978b). See also Loeblich et al. (1968) for original references prior to 1968 . The Miocene silicoflagellates from Sites 415 and 416 are shown on Plates 1, 2, and 3.

\section{COCCOLITHS}

Coccoliths are abundant and have slight to moderate overgrowth in the four cores. The middle-Miocene Sphenolithus heteromorphus Zone (Bramlette and Wilcoxon, 1967; Bukry, 1975) is represented by two samples: $415-4-5,63-65 \mathrm{~cm}(214 \mathrm{~m})$ and $416 \mathrm{~A}-1-2,7-8 \mathrm{~cm}$ $(148 \mathrm{~m})$. The presence of Cyclicargolithus floridanus, Cyclococcolithina macintyrei, Discoaster exilis, and Sphenolithus heteromorphus, together with the lack of Discoaster deflandrei and Helicosphaera ampliaperta, are the basis of the assignment. All of the other, deeper samples examined from Cores $415-5$ and 416A-2 contain $D$. deflandrei, $H$. ampliaperta, and $S$. heteromorphus, indicating the lower- or middle-Miocene Helicosphaera ampliaperta Zone (Bramlette and Wilcoxon, 1967; Bukry, 1975; Ryan et al., 1974). 


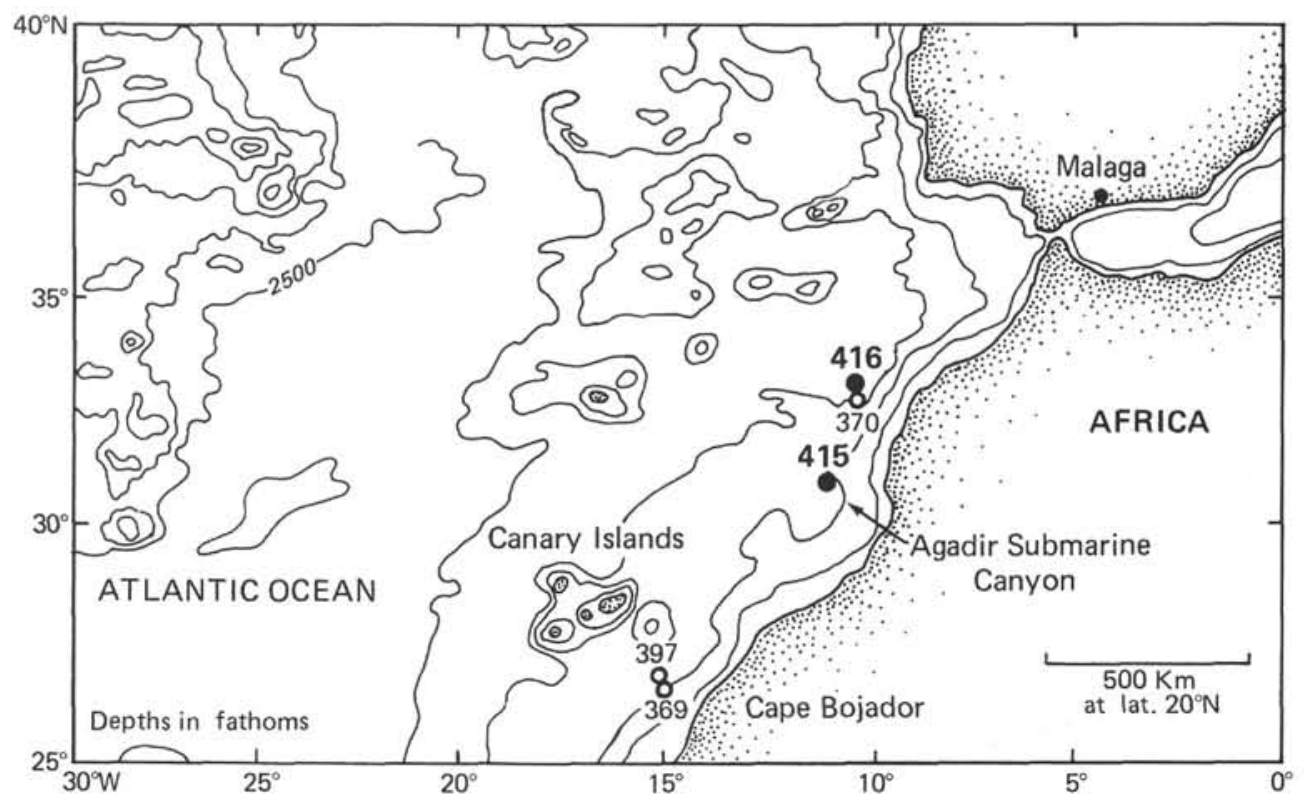

Figure 1. Locations of Sites 415 and 416, drilled during DSDP Leg 50. Nearby Sites 369 and 370 were drilled during Leg 41 and Site 397 during Leg 47.

\section{DIATOMS}

Diatoms are abundant and diverse in the four cores. They are slightly to moderately dissolved in all cores except Core $415-5$, in which the diatoms are well preserved. The co-occurrence and overlap of many distinctive species in the cores assures the correctness of correlation with the upper lower Miocene or lower middle Miocene. Diatom zonation of this interval is unsettled, because the relative ranges, and even the presence, of key guide fossils appear to be subject to ecologic conditions at the time of deposition (Abbott, 1978). This variability can be appreciated by comparing the occurrence patterns of such taxa as Actinocyclus ingens, A. lanceolatus, Annellus californicus, Cestodiscus spp., Coscinodiscus lewisianus, Craspedodiscus coscinodiscus, Denticula hustedtii, D. nicobarica, Raphidodiscus marylandicus, and species of the Rhaphoneis group between various regions (Kanaya, 1971; Bukry and Foster, 1973; Bukry, 1973, 1978b; Schrader, 1973a, 1974; Lohman, 1974; Gombos, 1975; Barron, 1976; Ernissee et al., 1977; Abbott, 1978). Differences between presumably coeval assemblages exist even at two closely spaced sites such as 415 and 416 (approximately $180 \mathrm{~km}$ apart). For example, short-ranged Annellus californicus and Raphidodiscus marylandicus occur together in Cores 415-5 and 416A-2, but Craspedodiscus coscinodiscus, which is common in $415-5$, is missing from 416A-2. Other differences, including the greater abundances of Paralia, Stephanopyxis, sponge spicules, and nonbiogenic clastics, suggest slightly shallower water at Site $\mathbf{4 1 6}$ during late early Miocene.

The taxonomy used to tabulate the selected species (Figure 3) comes variously from the following studies: Abbott, 1978; Andrews, 1974, 1975, 1976; Barron, 1976; Bukry and Foster, 1973; Ehrenberg, 1854; Gombos, 1975; Hanna, 1932; Kanaya, 1971; Kolbe, 1954; Lohman, 1948, 1974; Schrader, 1973a, 1973b, 1976;
Wornardt, 1967. Some of the Cestodiscus species in Sample 415-5-2, 58-60 cm (276 m) appear to approach the form of Actinocyclus ingens (see Kanaya, 1971; Lohman, 1974) (Plate 4, Figure 7). The Miocene diatoms from Leg 50 are shown on Plates 3, 4, and 5.

\section{CONCLUSIONS}

Diverse phytoplankton from the boundary between the lower and middle Miocene at DSDP Sites 415 and 416 permit correlation between diatom guide fossils $A n$ nellus californicus, Craspedodiscus coscinodiscus, Coscinodiscus lewisianus, Denticula hustedtii, D. nicobarica, and Raphidodiscus marylandicus and coccolith and silicoflagellate zones. A. californicus, D. lewisianus, and $D$. nicobarica occur in both the lower- or middleMiocene Helicosphaera ampliaperta Zone and the middle-Miocene Sphenolithus heteromorphus Zone. $C$. coscinodiscus and $R$. marylandicus occur only in the $H$. ampliaperta Zone and D. hustedtii only in the S. heteromorphus Zone. All the diatom species occur within the Corbisema triacantha Zone or Naviculopsis ponticula Zone of silicoflagellates. The naviculoid variant of Dictyocha brevispina ausonia occurs at the same stratigraphic level in the upper lower Miocene on both sides of the Atlantic Ocean and is not a local aberration. Concurrent treatment of phytoplankton groups from the same samples will help distinguish cosmopolitan and ecologically controlled ranges for silicoflagellate and diatom guide fossils.

\section{ACKNOWLEDGMENTS}

I thank John Barron, U.S. Geological Survey, for discussions on Miocene diatom ranges and Yves Lancelot, University of Paris, for authorization to study additional core materials from Sites 415 and 416 . The manuscript was improved by the reviews of Naja Mikkelsen, Scripps Institution of Oceanography, and John Barron, U.S. Geological Survey. 


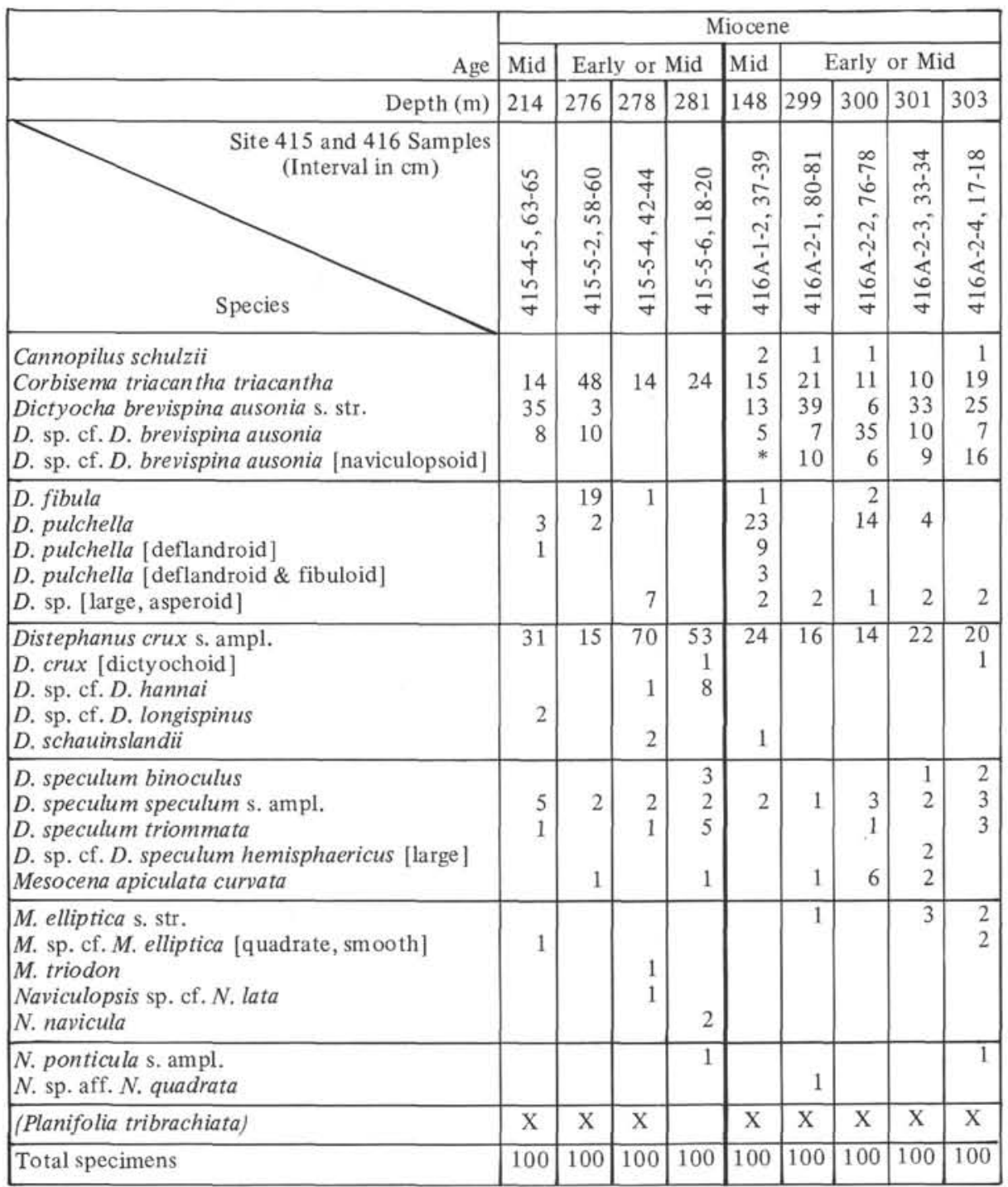

Figure 2. Silicoflagellates, recorded in per cent, from Miocene sediments in Cores 415-4 and 415-5 and Cores 416A-1 and 416A-2. All samples are assigned to the Corbisema triacantha Zone, except 415-5-6, 18-20 cm, which may belong to the Naviculopsis ponticula Zone. $*=$ specimens encountered after the count. $\mathrm{X}=$ presence of the endoskeletal dinoflagellate Planifolia tribrachiata (Ernissee, 1976).

\section{REFERENCES}

Abbott, W. H., 1978. Correlation and zonation of Miocene strata along the Atlantic margin of North America using diatoms and silicoflagellates, Marine Micropaleontology, v. 3 , p. $15-34$.

Andrews, G. W., 1974. Systematic position and stratigraphic significance of the marine Miocene diatom Raphidodiscus marylandicus Christian, Nova Hedwigia Beihefte, v. 45, p. 231-243.

1975. Taxonomy and stratigraphic occurrence of the marine diatom genus Rhaphoneis, Nova Hedwigia Beihefte, v. 53, p. 193-222.

1976. Miocene marine diatoms from the Choptank Formation, Calvert County, Maryland, U.S. Geol. Survey Prof. Paper 910, p. 26.
Barron, J. A., 1976. Revised Miocene and Pliocene diatom biostratigraphy of Upper Newport Bay, Newport Beach, California, Marine Micropaleontology, v. 1, p. 27-63.

Bramlette, M. N. and Wilcoxon, J. A., 1967. Middle Tertiary calcareous nannoplankton of the Cipero Section, Trinidad, W. I., Tulane Studies Geology, v. 5, p. 93-131.

Bukry, D., 1973. Phytoplankton stratigraphy, central Pacific Ocean, Deep Sea Drilling Project Leg 17. In Winterer, E. L., Ewing, J. I., et al., Deep Sea Drilling Project, Initial Reports of the Deep Sea Drilling Project, v. 17: Washington (U.S. Government Printing Office), p. 871-889. 1975. Coccolith and silicoflagellate stratigraphy, northwestern Pacific Ocean, Deep Sea Drilling Project Leg 32. In Larson, R. L., Moberly, R., et al., Initial Reports of the Deep Sea Drilling Project, v. 32: Washington (U.S. Government Printing Office), p. 677-701. 


\begin{tabular}{|c|c|c|c|c|c|c|c|c|c|}
\hline \multirow{3}{*}{$\begin{array}{r}\text { Age } \\
\text { Depth }(\mathrm{m})\end{array}$} & \multicolumn{9}{|c|}{ Miocene } \\
\hline & \multirow{2}{*}{$\frac{\text { Mid }}{214}$} & \multicolumn{3}{|c|}{ Early or Mid } & \multirow{2}{*}{\begin{tabular}{|l|} 
Mid \\
148
\end{tabular}} & \multicolumn{4}{|c|}{ Early or Mid } \\
\hline & & 276 & 278 & 281 & & 299 & 300 & 301 & 303 \\
\hline Species & 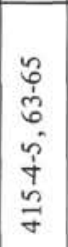 & 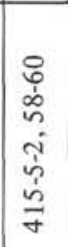 & 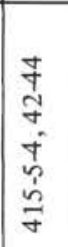 & 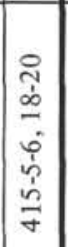 & 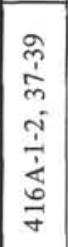 & 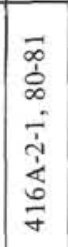 & 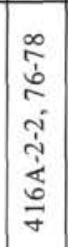 & 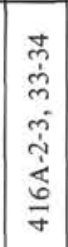 & 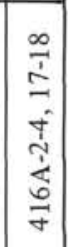 \\
\hline $\begin{array}{l}\text { Actinocyclus ehrenbergii } \\
\text { A. lanceolatus } \\
\text { Actinoptychus senarius } \\
\text { A. sp. cf. A. marylandicus } \\
\text { Annellus californicus }\end{array}$ & $\begin{array}{l}\mathrm{X} \\
\mathrm{x}\end{array}$ & $\begin{array}{l}\mathrm{X} \\
\mathrm{X} \\
\mathrm{X}\end{array}$ & $\begin{array}{l}\mathrm{X} \\
\mathrm{X} \\
\mathrm{X} \\
\mathrm{X}\end{array}$ & $\mathrm{X}$ & $\begin{array}{l}X \\
x\end{array}$ & $\mathrm{X}$ & $\begin{array}{l}X \\
X \\
X \\
X\end{array}$ & $\begin{array}{l}X \\
X \\
X\end{array}$ & $\begin{array}{l}X \\
X \\
X\end{array}$ \\
\hline $\begin{array}{l}\text { Asterolampra marylandicus [6-, 7-segments] } \\
\text { Asteromphalus sp. } \\
\text { Auliscus sp. A } \\
\text { Biddulphia spp. s. ampl. } \\
\text { Cestodiscus kugleri }\end{array}$ & $\mathrm{X}$ & $\begin{array}{l}\mathrm{m} \\
\mathrm{X}\end{array}$ & $\mathrm{X}$ & $\begin{array}{l}X \\
X\end{array}$ & $\mathrm{X}$ & $\mathrm{X}$ & $\mathrm{X}$ & $\begin{array}{l}\mathrm{X} \\
\mathrm{X} \\
\mathrm{X}\end{array}$ & $\mathrm{X}$ \\
\hline $\begin{array}{l}\text { C. spp. } \\
\text { Cocconeis spp. } \\
\text { Coscinodiscus lewisianus } \\
\text { Craspedodiscus coscinodiscus } \\
\text { Cussia paleacea }\end{array}$ & $\begin{array}{l}\mathrm{X} \\
\mathrm{X} \\
\mathrm{X}\end{array}$ & $\begin{array}{l}X \\
X \\
X\end{array}$ & $\begin{array}{l}X \\
X \\
X\end{array}$ & $\begin{array}{l}X \\
X\end{array}$ & $\begin{array}{l}\mathrm{X} \\
\mathrm{x}\end{array}$ & $\mathrm{X}$ & $\mathrm{X}$ & $\begin{array}{l}\mathrm{X} \\
\mathrm{X} \\
\mathrm{X} \\
\mathrm{X}\end{array}$ & $\begin{array}{l}x \\
x\end{array}$ \\
\hline $\begin{array}{l}\text { Denticula hustedtii } \\
\text { D. nicobarica } \\
\text { Diploneis sp. A } \\
\text { Eucampia balaustium } \\
\text { Liradiscus sp. cf. L. bipolaris }\end{array}$ & $\mathrm{X}$ & $\begin{array}{l}\text {. } \\
\mathrm{X} \\
\mathrm{X}\end{array}$ & $\begin{array}{l}\mathrm{X} \\
\mathrm{x}\end{array}$ & $\begin{array}{l}X \\
X\end{array}$ & $\begin{array}{l}X \\
x\end{array}$ & $\begin{array}{l}X \\
X\end{array}$ & & & $\begin{array}{l}X \\
X\end{array}$ \\
\hline $\begin{array}{l}\text { Paralia sulcata } \text { s. ampl. } \\
\text { Raphidodiscus marylandicus } \\
\text { Rhaphoneis amphiceros } \\
\text { R. diamantella } \\
\text { R. fossile }\end{array}$ & & $\begin{array}{l}X \\
X\end{array}$ & $\begin{array}{l}\mathrm{X} \\
\mathrm{X}\end{array}$ & $\begin{array}{l}X \\
\text { a }\end{array}$ & $\mathrm{X}$ & $\begin{array}{l}X \\
X\end{array}$ & $\mathrm{X}$ & $\mathrm{x}$ & $\begin{array}{l}\mathrm{E} \\
\mathrm{X} \\
\mathrm{X}\end{array}$ \\
\hline $\begin{array}{l}\text { R. gemmifera } \\
\text { R. sachalinensis } \\
\text { R.? sp. A } \\
\text { Sceptroneis grandis } \\
\text { Stephanogonia spp. }\end{array}$ & $\mathrm{X}$ & $\begin{array}{l}X \\
X \\
X\end{array}$ & $\mathrm{X}$ & $\mathrm{X}$ & $\mathrm{X}$ & $\mathrm{X}$ & $\begin{array}{l}X \\
X\end{array}$ & $\begin{array}{l}\mathrm{X} \\
\mathrm{X}\end{array}$ & $\mathrm{x}$ \\
\hline $\begin{array}{l}\text { Stephanopyxis spp. } \\
\text { Synedra jouseana } \\
\text { Xanthiopyxis cingulata } \\
X \text {. oblonga } \\
X . \text { ovalis }\end{array}$ & $\begin{array}{l}X \\
X\end{array}$ & $\begin{array}{l}X \\
X\end{array}$ & $\begin{array}{l}X \\
X\end{array}$ & $\begin{array}{l}\mathrm{X} \\
\mathrm{X} \\
\mathrm{X}\end{array}$ & $\begin{array}{l} \\
\mathrm{X} \\
\mathrm{X} \\
\mathrm{X}\end{array}$ & $\begin{array}{l}\mathbf{m} \\
\mathrm{X} \\
\mathrm{X} \\
\mathrm{X}\end{array}$ & $\begin{array}{l}\text { in } \\
X \\
X\end{array}$ & $\begin{array}{l}\text { प } \\
\mathrm{X} \\
\mathrm{X}\end{array}$ & $\mathrm{X}$ \\
\hline
\end{tabular}

Figure 3. Presence of selected diatom taxa in Miocene samples from Sites 415 and 416. $\mathrm{X}=$ present. Notable abundances of certain species are indicated (a).

1978a. Cenozoic coccolith and silicoflagellate stratigraphy, offshore northwest Africa, Deep Sea Drilling Project Leg 41. In Lancelot, Y., Seibold, E., et al., Initial Reports of the Deep Sea Drilling Project, v. 41: Washington (U.S. Government Printing Office), p. 689-708.

1978b. Cenozoic coccolith, silicoflagellate, and diatom stratigraphy, Deep Sea Drilling Project Leg 44. In Benson, W. E., Sheridan, R. E., et al., Initial Reports of the Deep Sea Drilling Project, v. 44: Washington (U.S. Government Printing Office), p. 807-863.

Bukry, D. and Foster, J. H., 1973. Silicoflagellate and diatom stratigraphy, Leg 16, Deep Sea Drilling Project. In van Andel, T., Heath, G. R., et al., Initial Reports of the Deep Sea Drilling Project, v. 16: Washington (U.S. Government Printing Office), p. 815-871.
Deflandre, G., 1941. Les notions de genre et de grade chez les Silicoflagellidées et la phylogenèse des mutants naviculaires, Acad. Sci. Comptes Rendus, v. 212, p. 100-102.

Ehrenberg, C. G., 1854. Mikrogeologie: Leipzig (Leopold Voss), p. 1-374.

Ernissee, J. J., 1976. Endoskeletal dinoflagellates from the Coosawhatchie Clay, Jasper County, South Carolina, South Carolina Div. Geology Geol. Notes, v. 20, p. 88-100.

Ernissee, J. J., Abbott, W. H., and Huddlestun, P. F., 1977. Microfossil correlation of the Coosawhatchie Clay (Hawthorne Formation, Miocene) of South Carolina, and its equivalent in Georgia, Marine Micropaleontology, v. 2, p. 105-119.

Gombos, A. M., Jr., 1975. Fossil diatoms from Leg 7, Deep Sea Drilling Project, Micropaleontology, v. 21, p. 306-333. 
Hanna, G. D., 1932. The diatoms of Sharktooth Hill, Kern County, California, California Acad. Sci. Proc., v. 20, p. 161-263.

Hyman, L. H., 1940. The Invertebrates: Protozoa through Ctenophora: New York (McGraw-Hill Book Co. Inc.).

Kanaya, T., 1971. Some aspects of pre-Quaternary diatoms in the oceans. In Funnel, B. M. and Riedel, W. R. (Eds.), The micropaleontology of oceans: Cambridge (Cambridge Univ. Press), p. 545-565.

Kolbe, R. W., 1954. Diatoms from equatorial Pacific cores, Swedish Deep-Sea Exped. Rept., v. 6, p. 3-49.

Ling, H. Y., 1972. Upper Cretaceous and Cenozoic silicoflagellates and ebridians, Am. Paleontology Bull., v. 62, p. 135-229.

Loeblich, A. R., 3rd, Loeblich, L. A., Tappan, H., and Loeblich, A. R., Jr., 1968. Annotated index of fossil and recent silicoflagellates and ebridians with descriptions and illustrations of validly proposed taxa, Geol. Soc. Am. Mem. 106.

Lohman, K. E., 1948. Middle Miocene diatoms from the Hammond Well, Maryland Dept. Geology, Mines and Water Resources Bull., v. 2, p. 151-333.

1974. Lower middle Miocene marine diatoms from Trinidad, Naturf. Gesell. Basel Verh., v. 84, p. 326-360.

Ryan, W. B. F., Cita, M. B., Dreyfus Rawson, M., Burckle, L. H., and Saito, T., 1974. A paleomagnetic assignment of Neogene stage boundaries and the development of isochronous datum planes between the Mediterranean, the Pacific and Indian Oceans in order to investigate the response of the world ocean to the Mediterranean "salinity crisis," Riv. Italiana Paleontologia, v. 80, p. 631-688.

Schrader, H.-J., 1973a. Cenozoic diatoms from the northeast Pacific, Leg 18. In Kulm, L. O., von Huene, R., et al., Initial Reports of the Deep Sea Drilling Project, v. 18: Washington (U. S. Government Printing Office), p. 673-797.

1973b. Stratigraphic distribution of marine species of the diatom Denticula in Neogene North Pacific sediments, Micropaleontology, v. 19, p. 417-430.

, 1974. Revised diatom stratigraphy of the experimental Mohole drilling, Guadalupe site, California Acad. Sci. Proc., v. 39, p. 517-562.

, 1976. Cenozoic planktonic diatom biostratigraphy of the southern Pacific Ocean. In Craddock, C., Hollister, C. D., et al., Initial Reports of the Deep Sea Drilling Project, v. 35: Washington (U.S. Government Printing Office), p. 605-671.

Schrader, H.-J. and Fenner, J., 1976. Norwegian Sea Cenozoic diatom biostratigraphy and taxonomy. In Talwani, M., Udintsev, G., et al., Initial Reports of the Deep Sea Drilling Project, v. 38: Washington (U.S. Government Printing Office), p. 921-1099.

Wornardt, W. W., 1967. Miocene and Pliocene marine diatoms from California, California Acad. Sci. Occasional Paper 63, p. 1-108. 
PLATE 1

Miocene Silicoflagellates from Sites 415 and 416

Figures 1-5, 7-16 magnified $800 \times$; scale bar equals $10 \mu \mathrm{m}$.

Figure 6 magnified $550 \times$; scale bar equals $10 \mu \mathrm{m}$.

Figures 1, 2 Cannopilus schulzii Deflandre.

1. Sample 416A-2-2, 76-78 cm (300 m).

2. Sample $416 \mathrm{~A}-1-2,37-39 \mathrm{~cm}(148 \mathrm{~m})$.

Figures 3, 4 Corbisema triacantha triacantha (Ehrenberg).

3. Sample 415-5-2, 58-60 cm (276 m).

4. Variant, Sample 415-5-6, 18-20 cm (281 m).

Figures 5, 6 Dictyocha brevispina ausonia (Deflandre).

5. Sample 416A-1-2, 37-39 cm (148 m).

6. Sample 416A-2-4, 17-18 cm (303 m).

Figures 7-14 Dictyocha sp. cf. D. brevispina ausonia (Deflandre) (naviculopsoid). Compare Dictyocha pons Ehrenberg.

7, 10. Sample 416A-2-2, 76-78 cm (300 m).

8, 9. Sample 416A-2-4, $17-18 \mathrm{~cm}(303 \mathrm{~m})$.

11, 14. Sample 416A-2-3, 33-34 cm (301 m).

12 , 13. Sample 416A-2-1, 80-81 cm (299 m).

Figure 15 Dictyocha sp. cf. D. brevispina ausonia (Deflandre). Intermediate to $D$. pulchella group.

Sample 415-5-2, 58-60 cm (276 m).

Figure 16 Dictyocha fibula Ehrenberg.

Long bar suggests affinity with $D$. brevispina ausonia group.

Sample 415-5-2, 58-60 cm (276 m). 
PLATE 1
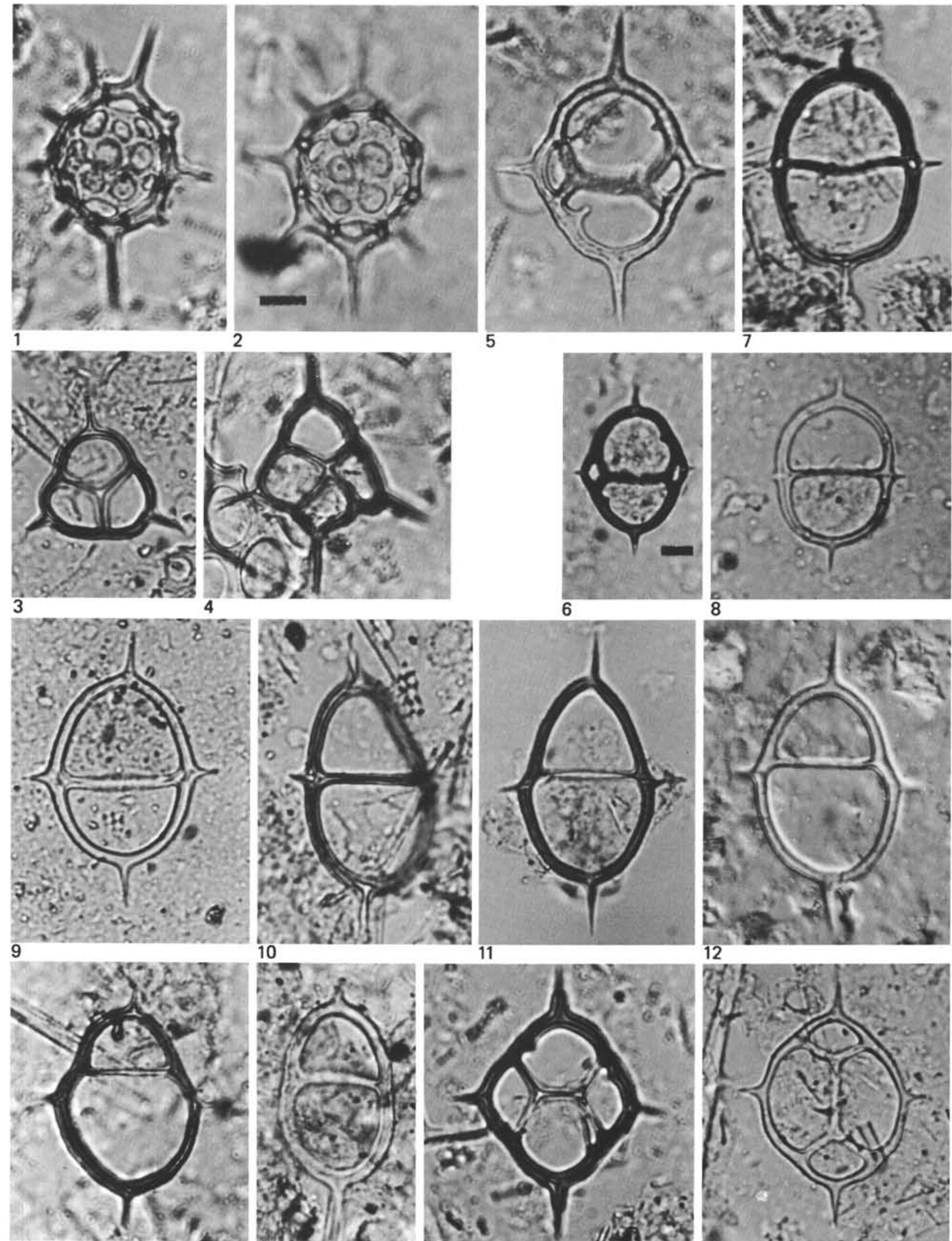

10

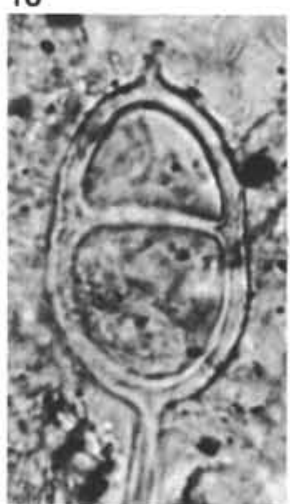

14

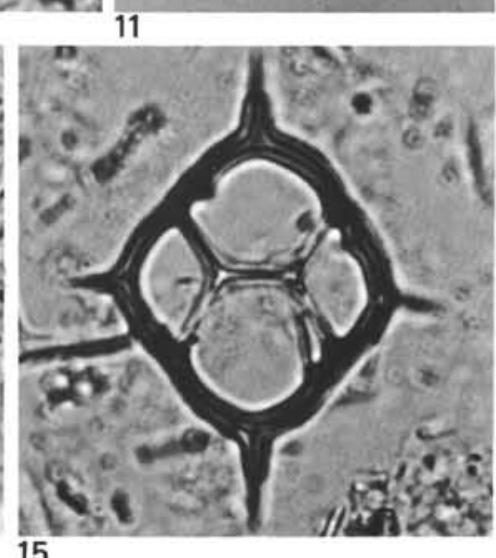

8
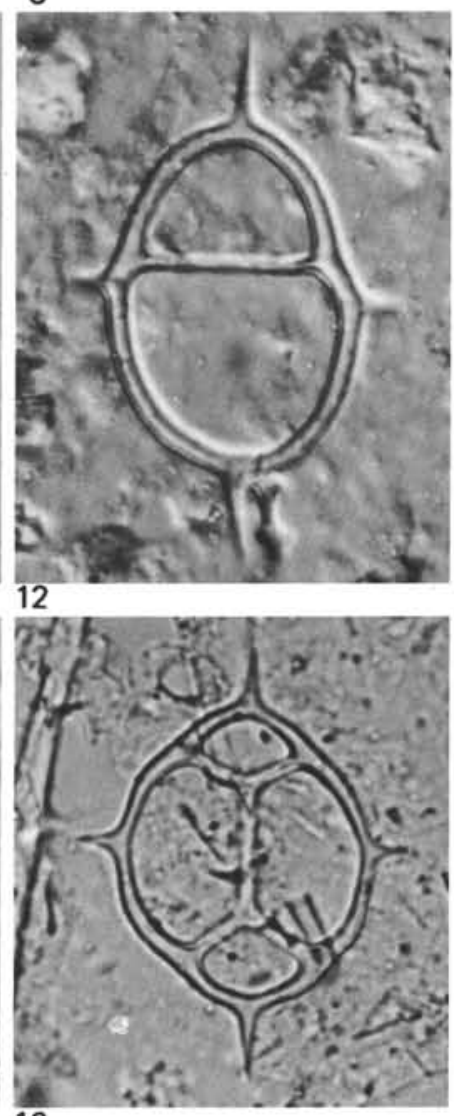


\section{PLATE 2}

Miocene Silicoflagellates from Sites 415 and 416

All figures magnified $800 \times$; scale bar equals $10 \mu \mathrm{m}$.

Figures 1, 2 Dictyocha fibula Ehrenberg.

Sample 415-5-2, 58-60 cm (276 m).

Figures 3-6 Dictyocha pulchella Bukry.

3. Sample $415-5-2,58-60 \mathrm{~cm}(276 \mathrm{~m})$.

4, 5. Sample 416A-1-2, 37-39 cm (148 m).

6. Deflandroid, Sample 416A-1-2, 37-39 cm (148 m).

Figure $7 \quad$ Dictyocha sp. (large, asperoid).

Scarce spineless form.

Sample 416A-2-2, 76-78 cm (300 m).

Figures 8, 9 Distephanus crux (Ehrenberg) s. ampl.

8. Sample 416A-2-4, $17-18 \mathrm{~cm}(303 \mathrm{~m})$.

9. Sample $415-5-6,18-20 \mathrm{~cm}(281 \mathrm{~m})$.

Figures 10, 11 Distephanus sp. cf. D. hannai (Bukry).

Sample 415-5-6, 18-20 cm (281 m).

Figure 12 Distephanus speculum speculum (Ehrenberg) s. ampl.

Sample 415-5-2, 58-60 cm (276 m).

Figure 13 Distephanus speculum triommata (Ehrenberg). Sample 415-5-4, 42-44 cm (278 m).

Figures 14, 15 Mesocena apiculata curvata Bukry.

14. Sample 415-5-2, 58-60 cm (276 m).

15. Sample 416A-2-3, 33-34 cm (301 m).

Figure $16 \quad$ Mesocena elliptica (Ehrenberg).

Sample 416a-2-3, 33-34 cm (301 m). 
PLATE 2
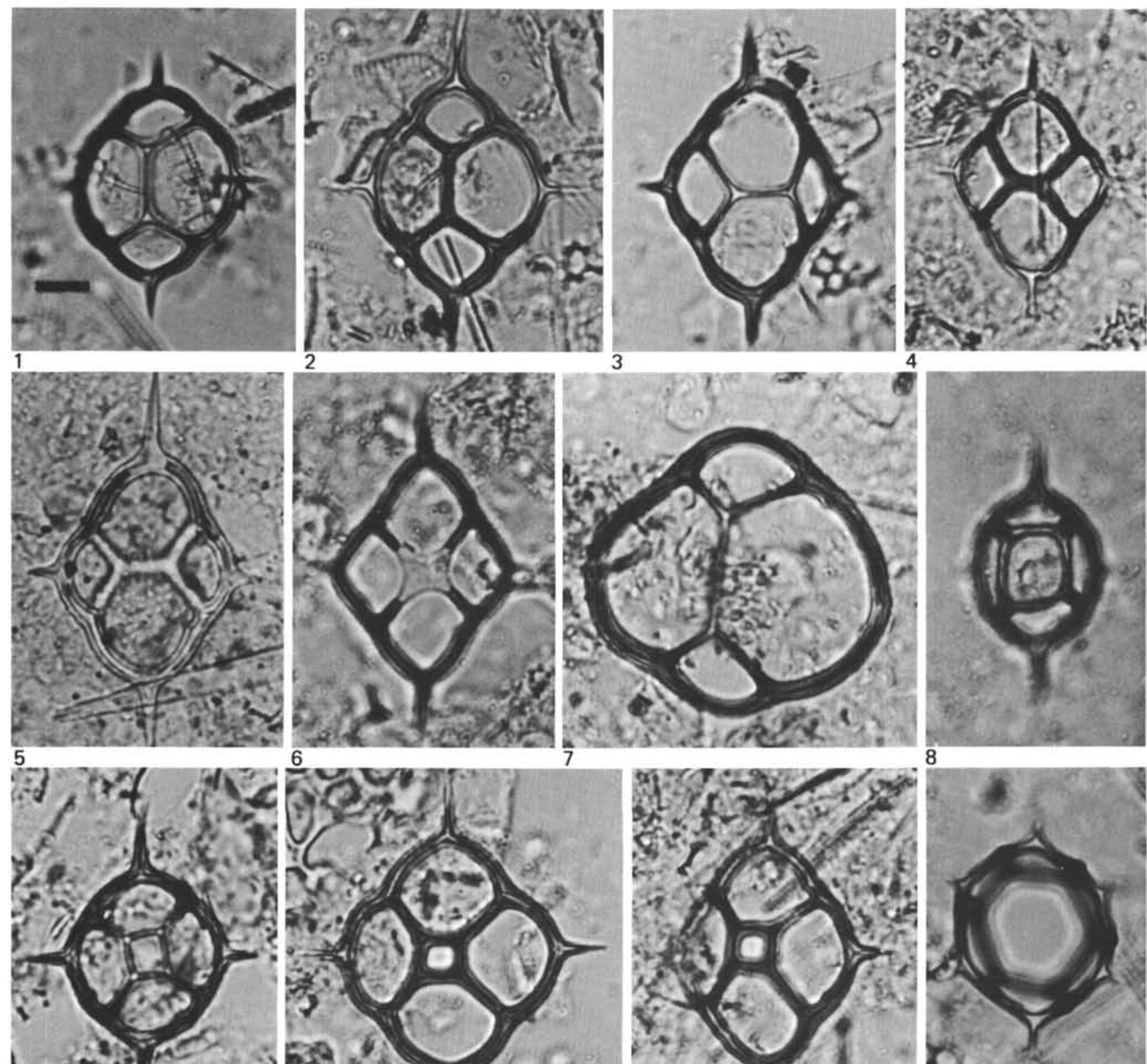

$\rightarrow$ anc

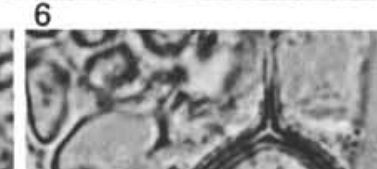

Han $x$.

1.
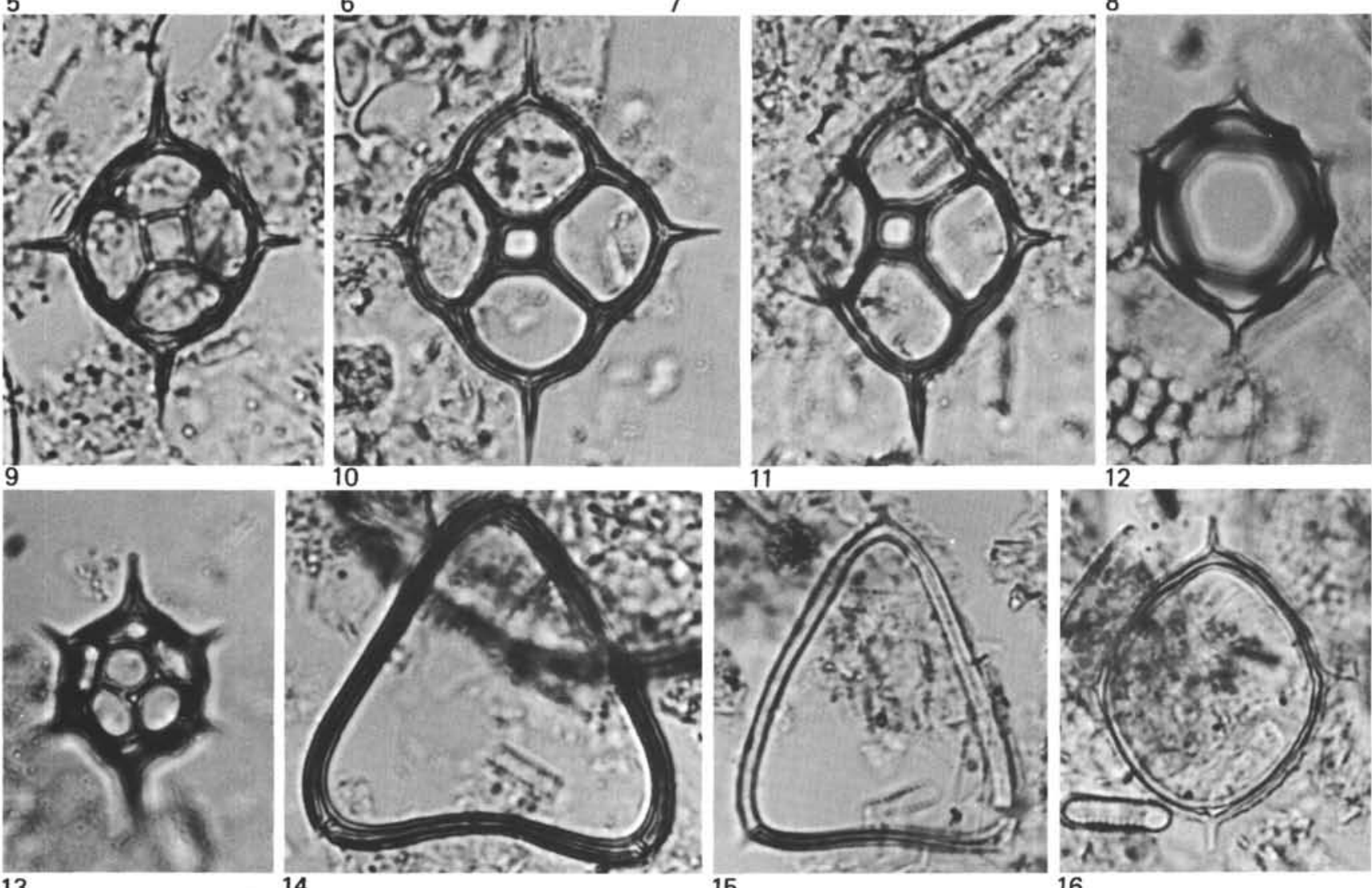
PLATE 3

Miocene Silicoflagellates and Diatoms from Sites 415 and 416 Figure 8 magnified, $1050 \times$; scale bar equals $10 \mu \mathrm{m}$.

Figures 1-7, 9-12 magnified $800 \times$; scale bar equals $10 \mu \mathrm{m}$.

Figures 1-3 Naviculopsis ponticula (Ehrenberg).

1. Normal, Sample 415-5-6, 18-20 cm (281 m).

2. Spined, Sample 416A-2-4, 17-18 cm (303 m).

3. Variant, Sample $415-5-6,18-20 \mathrm{~cm}(281 \mathrm{~m})$.

Figures 4, $5 \quad$ Naviculopsis navicula (Ehrenberg).

4. Normal, Sample 415-5-6, 18-20 cm (281 m).

5. Variant, Sample 416A-2-1, 30-31 cm (298 m).

Figure $6 \quad$ Actinocyclus ehrenbergii Ralfs.

Sample 416A-2-4, 17-18 cm (303 m).

Figure 7 Actinocyclus lanceolatus (Castracane).

Sample 415-5-2, 58-60 cm (276 m).

Figure 8 Actinoptychus sp. cf. A. marylandicus Andrews. Sample 416A-2-4, 17-18 cm (303 m).

Figures 9, 10 Annellus californicus Tempere.

Sample 415-5-2, 58-60 cm (276 m).

Figure 11 Asteromphalus sp.

Sample 416A-2-3, 33-34 cm (301 cm).

Figure 12 Auliscus sp. A.

Sample 415-5-2, 58-60 cm (276 m). 
PLATE 3
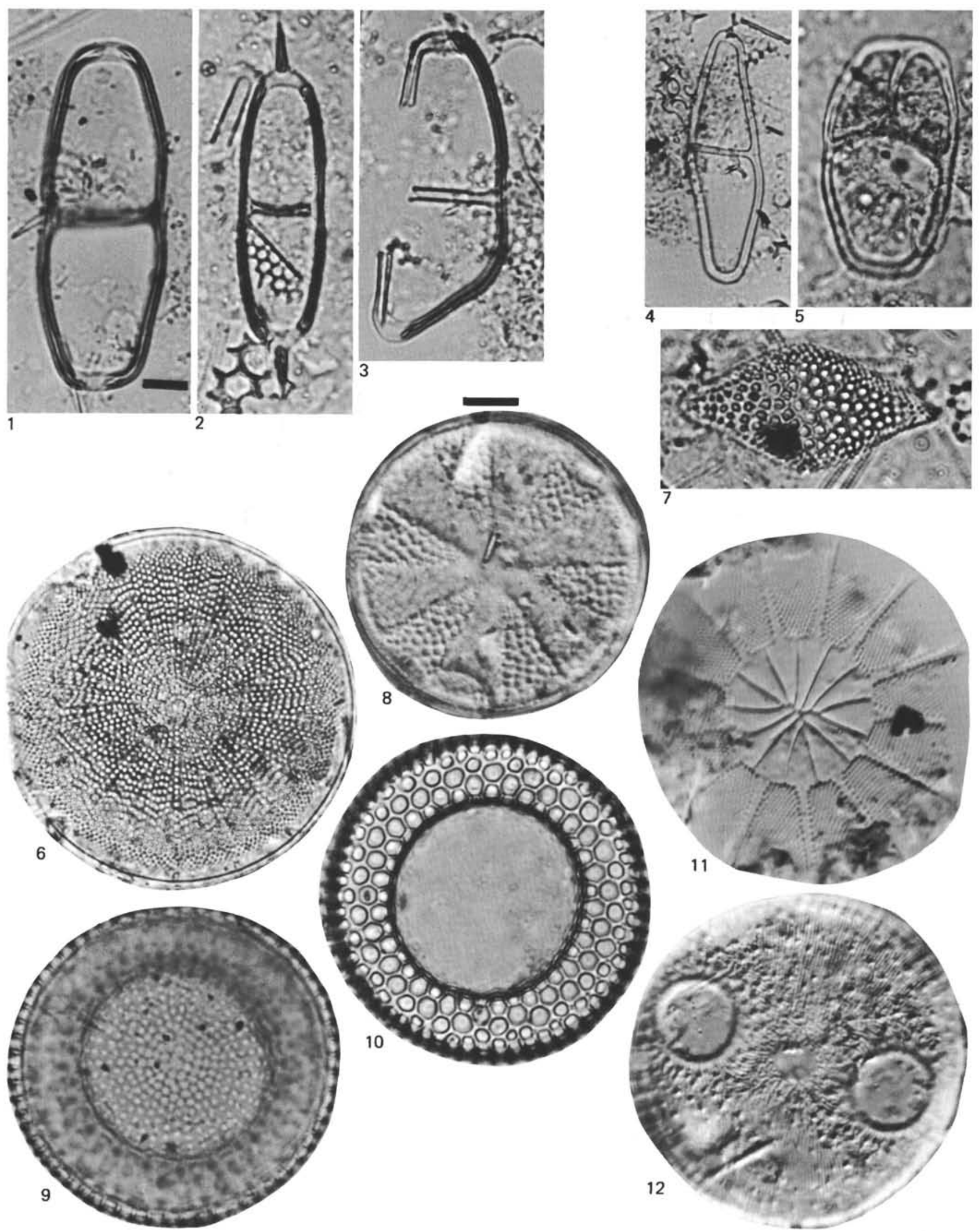
PLATE 4

Miocene Diatoms from Sites 415 and 416

Figures 5-7, 13, 14 magnified $1050 \times$; scale bar equals $10 \mu \mathrm{m}$.

Figures $1-4,8-12,15$ magnified $800 \times$; scale bar equals $10 \mu \mathrm{m}$.

Figures 1, $2 \quad$ Biddulphia sp. s. ampl.

1. Sample 416A-2-1, 80-81 cm (299 m).

2. Sample $416 \mathrm{~A}-1-2,37-39 \mathrm{~cm}(148 \mathrm{~m})$.

Figures 3, 4 Bruniopsis? sp. fragments. Sample 415-5-6, 18-20 $\mathrm{cm}(281 \mathrm{~m})$.

Figures 5, 6 Cestodiscus kugleri Lohman.

5. Sample 415-5-2, 58-60 cm (276 m).

6. Sample $415-5-6,18-20 \mathrm{~cm}(281 \mathrm{~m})$.

Figures 7-11 Cestodiscus spp.

7. Mimic of Actinocyclus ingens, Sample 415$5-2,58-60 \mathrm{~cm}(276 \mathrm{~m})$.

8. Sample 416A-2-3, 33-34 cm (301 m).

9,10 . Low and high focus, Sample 415-5-2, 58$60 \mathrm{~cm}(276 \mathrm{~m})$.

11. Sample 415-5-2, 58-60 cm (276 m).

Figures 12, 13 Cocconeis spp.

12. Sample $416 \mathrm{~A}-2-3,33-34 \mathrm{~cm}(301 \mathrm{~m})$.

13. Sample 415A-4-5, 63-65 cm (214 m).

Figures 14-16 Coscinodiscus lewisianus Greville.

14,16 . Sample $415-5-2,58-60 \mathrm{~cm}(276 \mathrm{~m})$.

15. Sample 415-5-4, $42-44 \mathrm{~cm}(278 \mathrm{~m})$. 
PLATE 4
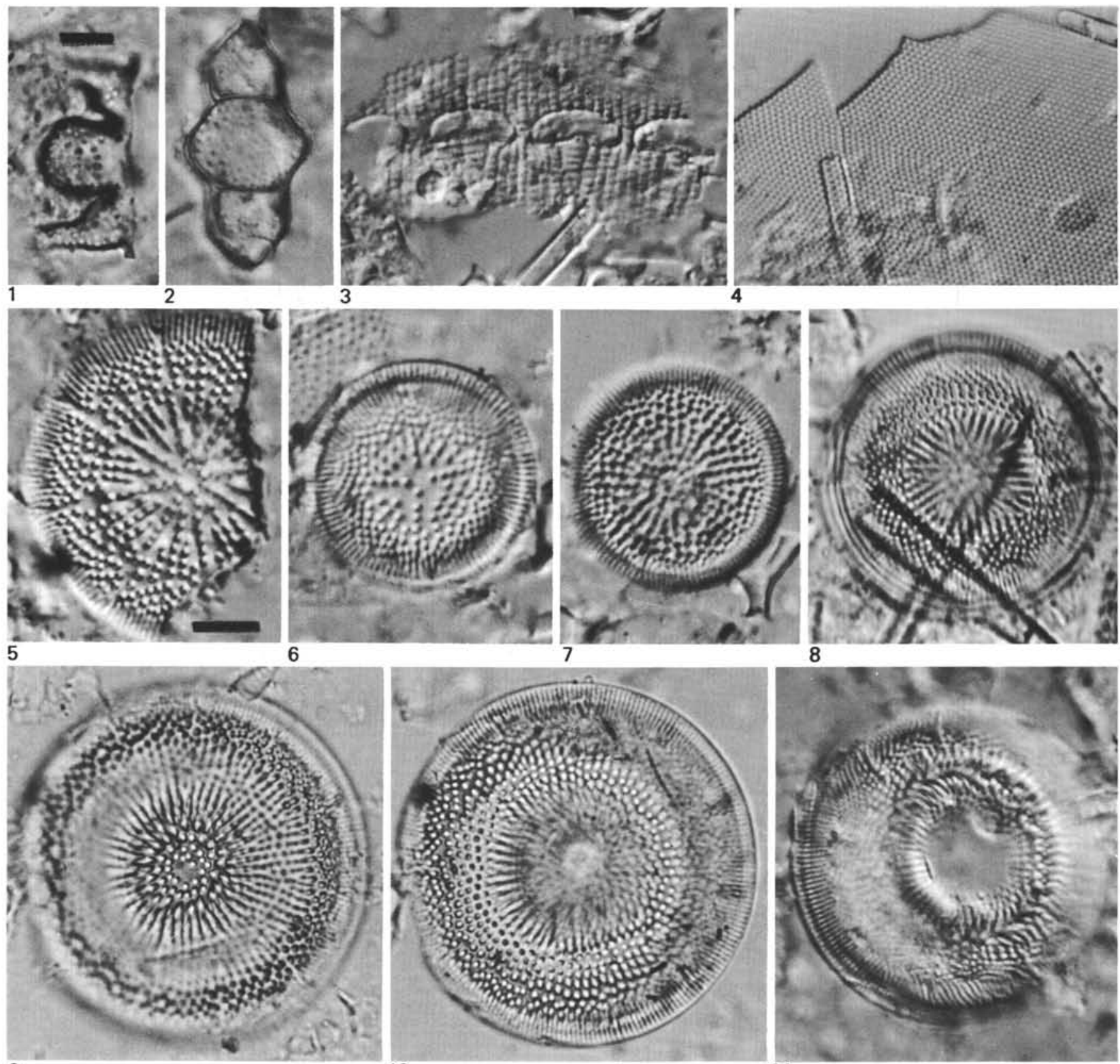

9

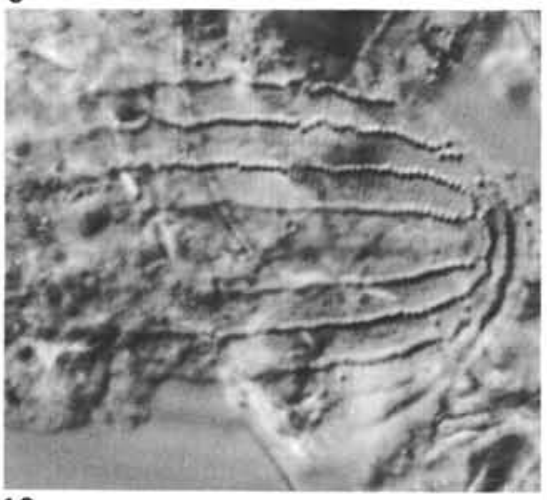

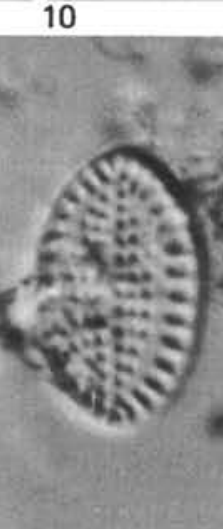

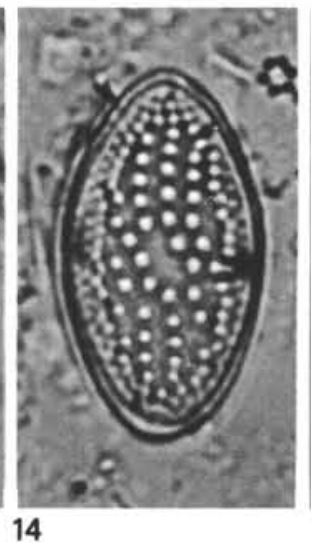

11

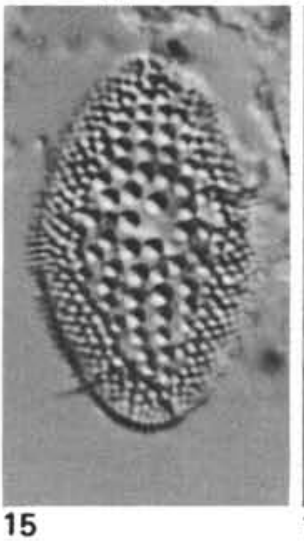

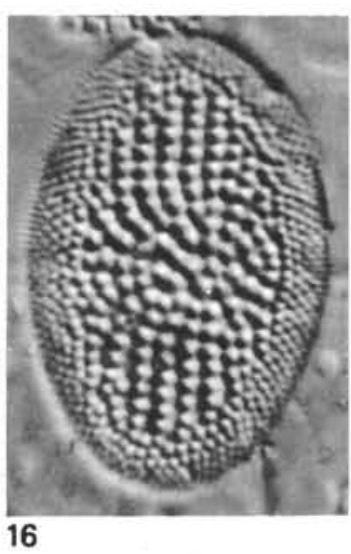




\section{PLATE 5}

Miocene Diatoms from Sites 415 and 416

Figures $4,5,8-10,15,16,18$ magnified $1050 \times$; scale bar equals $10 \mu \mathrm{m}$.

Figures 3, 6, 7, 11, 13, 14, 17 magnified $800 \times$; scale bar equals $10 \mu \mathrm{m}$

Figures 1, 2, 12 magnified $350 \times$; scale bar equals $20 \mu \mathrm{m}$.

Figures 1, 2 Craspedodiscus coscinodiscus Ehrenberg. Sample 415-5-2, 58-60 cm (276 m).

Figure $3 \quad$ Cussia paleacea (Grunow).

Sample 415-5-2, 58-60 cm (276 m).

Figures 4, 5 Denticula nicobarica Grunow.

Sample 415-5-2, 58-60 cm (276 m).

Figure 6 Diploneis sp. A

Sample 416A-2-4, 17-18 cm (303 m).

Figures 7, 8 Eucampia balaustium Castracane.

7. Sample 415-5-6, $18-20 \mathrm{~cm}(281 \mathrm{~m})$.

8. Sample 415-5-2, 58-60 cm (276 m).

Figure 9 Hyalodiscus sp.

Sample 416A-2-4, 17-18 cm (303 m).

Figure $10 \quad$ Liradiscus sp. cf. L. bipolaris Lohman.

Sample 415-5-2, 58-60 cm (276 m).

Figure $11 \quad$ Pseudopyxilla sp. cf. $P$. directa (Pantocsek).

Sample 415-4-5, 63-65 cm (214 m).

Figure $12 \quad$ Pseudopyxilla sp. cf. P. dubia (Grunow).

Sample 416A-2-4, 17-18 cm (303 m).

Figure $13 \quad$ Pseudopyxilla sp.

Sample 415-5-6, 18-20 cm (281 m).

Figures 14-18 Raphidodiscus marylandicus Christian.

14. Sample $415-5-2,58-60 \mathrm{~cm}(276 \mathrm{~m})$.

15. Isolated central area, Sample 415-5-6, 18-20 $\mathrm{cm}(281 \mathrm{~cm})$.

16. Frustule, Sample $415-5-2,58-60 \mathrm{~cm}(276 \mathrm{~m})$.

17 , 18. Sample 415-5-2, 58-60 cm (276 m).

Figure 19 Rhaphoneis amphiceros (Ehrenberg).

Sample 416A-2-4, 17-18 cm (303 m). 
PLATE 5

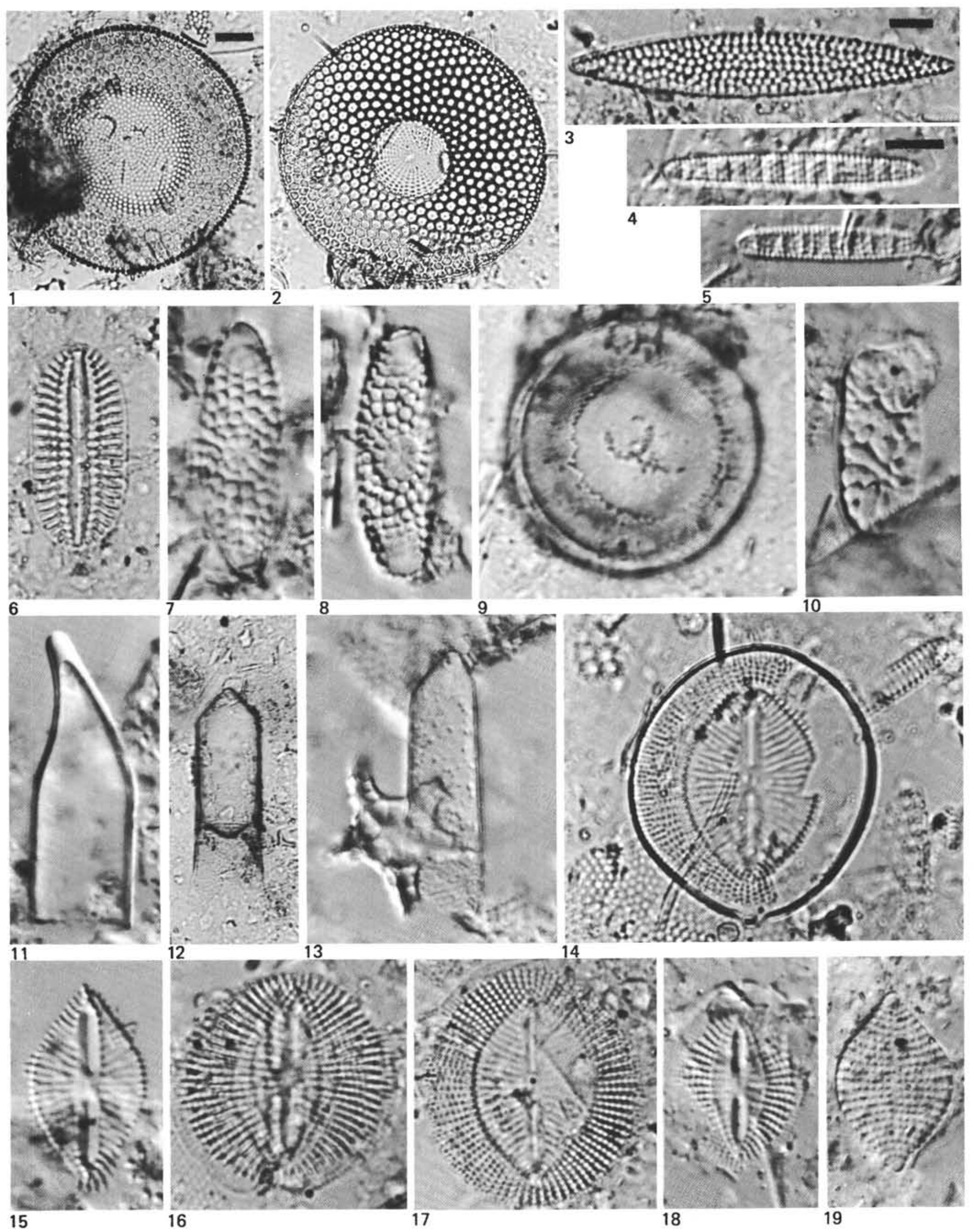




\section{PLATE 6}

Miocene Diatoms and Planifolia from Sites 415 and 416.

Figures 3, 7-10, 12, 14, 15 magnified $1050 \times$; scale bar equals $10 \mu \mathrm{m}$.

Figures 1, 2, 4-6, 11, 13 magnified $800 \times$; scale bar equals $10 \mu \mathrm{m}$.

Figures 1,2 Rhaphoneis diamantella Andrews. Sample 415-5-6, 18-20 cm (281 m).

Figures 3-5 Rhaphoneis fossile (Grunow).

3, 4. Sample 416A-2-4, 17-18 cm (303 m).

5. Sample 416A-2-2, 76-78 cm (300 m).

Figure $6 \quad$ Rhaphoneis gemmifera Ehrenberg. Sample 415-5-4, 42-44 cm (278 m).

Figures 7, 8 Rhaphoneis sachalinensis Sheshukova-Poretskaya.

7. Sample 416A-2-2, 76-78 cm (300 m).

8. Sample $415-5-2,58-60 \mathrm{~cm}(276 \mathrm{~m})$.

Figures 9, 10 Rhaphoneis? sp. A.

Sample 415-5-2, 58-60 cm (276 m).

Figure $11 \quad$ Stephanogonia sp.

Sample 415-5-6, 18-20 cm (281 m).

Figure 12 Xanthiopyxis cingulata Ehrenberg. Sample 415-5-6, 18-20 cm (281 m).

Figure 13 Xanthiopyxis oblonga Ehrenberg. Sample 416A-1-2, 37-39 cm (148 m).

Figures 14, 15 Planifolia tribrachiata Ernissee.

14. Sample 416A-2-4, $17-18 \mathrm{~cm}$ (303 m).

15. Sample $415-5-2,58-60 \mathrm{~cm}(276 \mathrm{~m})$. 
PLATE 6
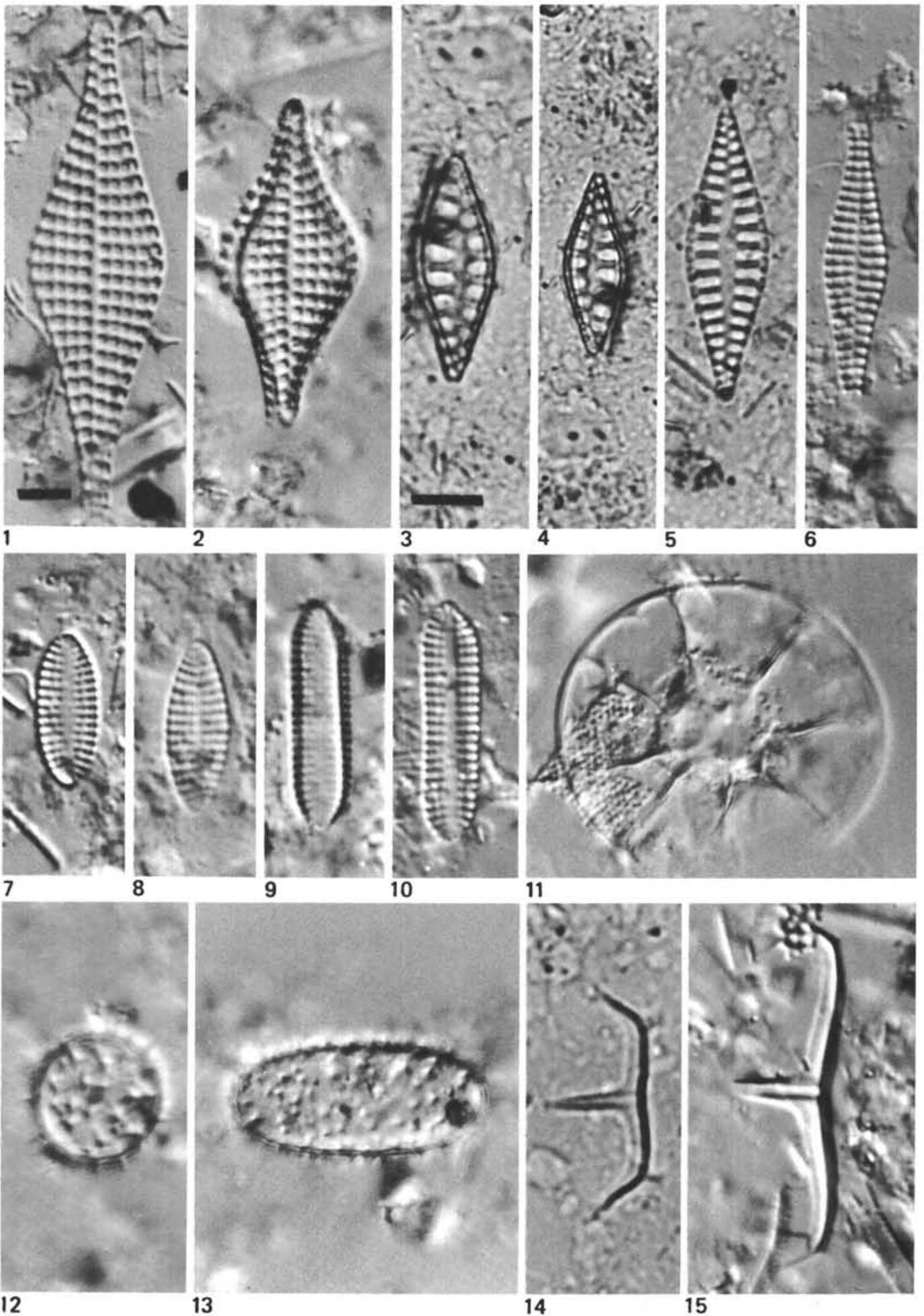

15 